\title{
NUMERICAL SOLUTIONS FOR CONVECTIVE BOUNDARY LAYER FLOW OF MICROPOLAR JEFFREY FLUID WITH PRESCRIBE WALL TEMPERATURE
}

\author{
Noraihan Afiqah Rawi ${ }^{1}$, Nor Athirah Mohd Zin ${ }^{1}$, Asma Khalid ${ }^{2}$, \\ Abdul Rahman Mohd Kasim ${ }^{3}$, Zaiton Mat Isa ${ }^{1}$, Sharidan Shafie ${ }^{1}$ \\ ${ }^{1}$ Department of Mathematical Sciences, Faculty of Science, Universiti \\ Teknologi Malaysia, 81310 UTM Johor Bahru, Johor, Malaysia, \\ noraihanafiqah@utm.my, norathirah24@gmail.com, zaitonmi@utm.my, \\ sharidan@utm.my \\ ${ }^{2}$ Department of Mathematics, SBK Womens University, Quetta 87300, \\ Pakistan, awaisiasma@gmail.com \\ ${ }^{3}$ Faculty of Industrial Sciences \& Technology, Universiti Malaysia Pahang, \\ Lebuhraya Tun Razak, 26300 Gambang, Kuantan, Pahang, Malaysia, \\ rahmanmohd@ump.edu.my
}

\begin{abstract}
The steady two dimensional convective boundary layer flow of micropolar Jeffrey fluid past a permeable stretching sheet is studied in this paper. The governing boundary layer equation in the form of partial differential equations are transformed into nonlinear coupled ordinary differential equations and solved numerically using an implicit finite-difference scheme known as Keller-box method. The effect of Prandtl number, Deborah number and material parameter specifically for strong concentration of microelements on the velocity, microrotation and temperature profiles as well as skin friction and heat transfer coefficients are presented and discussed. An excellent agreement is observed between the present and earlier published results for some special cases. The results revealed that, the effect of Deborah number and stretching parameter are increased the heat transfer coefficient while the opposite trend is observed for the effects of material and velocity slip parameters. It was also observed that, the values of skin friction increased with the increment on the values of all studied parameters.
\end{abstract}

Key words and Phrases: Boundary layer flow, Micropolar Jeffrey fluid, Keller-box, Numerical solution.

2020 Mathematics Subject Classification: 76-10, 35Q35, 37M05 Received: 14-07-2017, accepted: 03-07-2019. 


\section{INTRODUCTION}

Analysis of non-Newtonian fluids is still a topic of great interest. Scientists have stimulated in this field of research due to the numerous applications of nonNewtonian fluids in pharmaceuticals, physiology, fiber technology, food products, coating of wires, crystal growth etc. Such fluids have a nonlinear relationship between the stress and the rate of strain at a point and exhibit some worth notice facts which are due to their elastic nature. Many materials of industrial significance, notably polymer systems (melt and solutions) and multi-phase system such as foams, emulsions, and slurries, which involve a range of non-Newtonian characteristic including shear thinning/shear thickening, shear-dependent viscosity, stress relaxation, and normal stress difference. Hence, due to the practical and fundamental association of non-Newtonian fluids to industrial applications, several studies $[1,2,3,4,5,6,7,8,9,10]$ of these fluids in different geometries have been carried out.

Recently, there are many types of non-Newtonian fluids which have become very popular in the literature such as micropolar fluid, second grade fluid, OldroydB fluid, Jeffrey fluid and Casson fluids. Among these, Jeffrey fluid has been proved relatively as the simpler viscoelastic non-Newtonian fluid which exhibits the shear thinning characteristics, yield stress and high shear viscosity [11]. Originally, this rheological model was introduced by Jeffreys [12] to stimulate the earth crustal flow problems. This model can be degenerates to a Newtonian fluid at a very high wall shear stress i.e. when the wall stress is much greater than yield stress. Several recent contributions dealing with the flow of Jeffrey fluid under different effects and geometry have been studied by many researchers.

Nadeem et al. [13] presented the peristaltic flow of a Jeffrey fluid with variable viscosity in an asymmetric channel. Some unsteady flows of a Jeffrey fluid between two side walls over a plane wall has been reported by Khan et al. [14], whereas Hayat et al. [15] discussed the boundary layer flow of a Jeffrey fluid with convective boundary conditions. Thermal radiation effect on the mixed convection stagnationpoint flow in a Jeffery fluid and the effects of an endoscope and magnetic field on the peristalsis involving Jeffrey fluid are also presented by Hayat et al. $[16,17]$. On the other hand, Vajravelu et al. [18] studied the influence of heat transfer on the peristaltic transport of Jeffrey fluid in a vertical porous stratum. Turkyilmazoglu and Pop [19] obtained exact analytical solutions for the flow and heat transfer near the stagnation point on a stretching/shrinking sheet in a Jeffrey fluid. Recently, Zin et al. [20] discussed the influence of Newtonian heating and thermal radiation on the combined heat and mass transfers for the unsteady free convection MHD flow of Jeffrey fluid past an oscillating vertical plate.

Even there are a lot of papers studied regarding the Jeffrey fluid, but the solutions in term of numerical are still few and the solution on this type of fluid with microrotation effect still hard to find. Basically, micropolar fluid is a subset of the micromorphic fluid theory, which first introduced by Eringen [21]. This fluid are theoretically represent fluids that contain rigid randomly oriented particles suspended in viscous medium which have an essential micro motion in rotation. 
In real life, the fluids that can be classified as micropolar fluid are colloidal fluids, biological fluids in thin vessels such as human or animal bloods and also polymeric suspensions. Independent studies of micropolar fluid without Jeffrey fluid term (called Deborah number) have been done by many researchers in past few decades $[22,23,24,25,26,27,28,29,30]$.

Motivated by the above literature, this article presents the numerical solutions for the problem of convective boundary layer flow of micropolar Jeffery fluid with prescribe wall temperature. The governing equations are first transformed into ordinary differential equations using appropriated similarity transformation and then solved by using Keller box method. The solutions of fluid characteristics in term of skin friction and heat transfer coefficient as well as velocity and temperature distributions with various values of studied parameters which are Deborah number, Prandtl number, material parameter, stretching parameter and slip velocity parameter are presented graphically and in tabular form.

\section{PROBLEM FORMULATION}

Consider the steady two dimensional boundary layer stagnation point of an incompressible Jeffrey fluid past a stretching sheet with prescribed wall temperature. The positive $x$-coordinate is the distance measured along the sheet and the positive $y$-coordinate measures the distance normal to the sheet. It is assumed that, the temperature of the sheet varies linearly with the distance $x$ along the sheet, where $T_{w}(x)>T_{\infty}$ with $T_{w}(x)$ being the temperature of the sheet and $T_{\infty}$ being the uniform temperature of the ambient fluid. In this problem, the effect of microrotation is also considered. The constitutive equation of Jeffrey fluid are given by

$$
\begin{gathered}
\mathbf{T}=-p \mathbf{I}+\mathbf{S}, \\
\left.\mathbf{S}=\frac{\mu}{1+\lambda_{1}} \overline{(\gamma}+\lambda_{2} \bar{\gamma}\right) .
\end{gathered}
$$

Here, $\mathbf{T}$ is the Cauchy stress tensor, $-p \mathbf{I}$ is the indeterminate part of stress, $\mathbf{S}$ is the extra stress tensor, $\mu$ is the coefficient of viscosity, $\lambda_{1}$ is the ratio of relaxation to retardation times and $\lambda_{2}$ is the retardation time respectively, $\bar{\gamma}$ is the shear rate and dots over the quantities indicate differentiation with respect to time. It is worth to mention that, if $\lambda_{1}=\lambda_{2}=0$, the equation (2) reduces to the expressions of an incompressible viscous fluid. Under the Boussinesq and boundary layer approximations, the governing equations of momentum, microrotation and energy for Jeffrey fluid are written as

$$
\frac{\partial u}{\partial x}+\frac{\partial v}{\partial y}=0
$$




$$
\begin{gathered}
u \frac{\partial u}{\partial x}+v \frac{\partial u}{\partial y}=\frac{\left(\nu+\frac{\kappa}{\rho}\right)}{1+\lambda_{1}}\left[\frac{\partial^{2} u}{\partial y^{2}}+\lambda_{2}\left(u \frac{\partial^{3} u}{\partial x \partial y^{2}}-\frac{\partial u}{\partial x} \frac{\partial^{2} u}{\partial y^{2}}+\frac{\partial u}{\partial y} \frac{\partial^{2} u}{\partial x \partial y}+v \frac{\partial^{3} u}{\partial y^{3}}\right)\right]+ \\
u_{e} \frac{\partial u_{e}}{\partial x}+\frac{\kappa}{\rho} \frac{\partial N}{\partial y} \\
u \frac{\partial N}{\partial x}+v \frac{\partial N}{\partial y}=\frac{\gamma}{\rho j} \frac{\partial^{2} N}{\partial y^{2}}-\frac{\kappa}{\rho j}\left(2 N+\frac{\partial u}{\partial y}\right) \\
u \frac{\partial T}{\partial x}+v \frac{\partial T}{\partial y}=\frac{k^{*}}{\rho c_{p}} \frac{\partial^{2} T}{\partial y^{2}}
\end{gathered}
$$

The appropriate physical boundary conditions are given by

$$
\begin{aligned}
& u=c x+g_{x} \frac{\partial u}{\partial y}, \quad v=0, \quad N=-n \frac{\partial u}{\partial y}, \quad T=T_{w}, \quad \text { at } y=0, \\
& u=u_{e} \rightarrow a x, \quad N \rightarrow 0, \quad \frac{\partial u}{\partial y} \rightarrow 0, \quad T \rightarrow T_{\infty}, \quad \text { as } y \rightarrow \infty,
\end{aligned}
$$

where $u$ and $v$ are the velocity components along the $x$ and $y$-axes, $\nu$ is the kinematic viscosity, $\kappa$ is a vortex viscosity, $\rho$ is the fluid density, $u_{e}$ is the velocity outside the boundary layer, $N$ is the component of microrotation vector normal to the $x$ and $y$-axes, $\gamma$ the spin gradient, $j=\nu / a$ is the microinertia density, $a$ is an arbitrary constants, $T$ is the temperature, $k^{*}$ is the thermal conductivity, $c_{p}$ is the specific heat capacity, $c$ is an arbitrary constants, $g_{x}$ is slip constant depending on the $\lambda_{1}$ and $n$ is the concentration of microelements in the range of $0 \leq n \leq 1$ (see Lok [25]), respectively. Here, the case of $n=0$ is called strong concentration indicated $N=0$ near the wall, represents the concentrated particle flows in which the microelements close to the wall surface are unable to rotate [31] while, $n=1 / 2$ is called weak concentration and represent the vanishing of the anti-symmetric part of stress tensor [32]. Following Rees and Bassom [33] and Lok [34], $\gamma$ is defined as

$$
\gamma=\left(\mu+\frac{\kappa}{2}\right) j=\mu\left(1+\frac{K}{2}\right) j
$$

where $\mu$ is a dynamic viscosity and $K=\kappa / \mu$ is denoted as material parameter. Equation (8) is invoked to allow the field of equations to predict the correct behaviour in the limiting case when the microstructure effects become negligible, and the total spin $N$ reduces to the angular velocity [32]. The complexity of the problem is reduced by introducing the following similarity transformations,

$$
\begin{gathered}
\eta=y \sqrt{\frac{c\left(1+\lambda_{1}\right)}{\nu}}, \quad u=c x \frac{\partial f}{\partial \eta}, \quad v=-\sqrt{\frac{c \nu}{1+\lambda_{1}}} f(\eta), \\
N=c x \sqrt{\frac{c\left(1+\lambda_{1}\right)}{\nu}} g(\eta), \quad \theta=\frac{T-T_{\infty}}{T_{w}-T_{\infty}} .
\end{gathered}
$$

Substitution of the equation (9) into equation (3) until equation (6) give, 


$$
\begin{gathered}
f(\eta) \frac{\partial^{2} f}{\partial \eta^{2}}-\left[\frac{\partial f}{\partial \eta}\right]^{2}+(1+K)\left[\frac{\partial^{3} f}{\partial \eta^{3}}+\beta\left[\left(\frac{\partial^{2} f}{\partial \eta^{2}}\right)^{2}-f(\eta) \frac{\partial^{4} f}{\partial \eta^{4}}\right]\right]+K \frac{\partial g}{\partial \eta}+\delta^{2}=0 \\
\left(1+\frac{K}{2}\right) \frac{\partial g^{2}}{\partial \eta}+f \frac{\partial g}{\partial \eta}-\frac{\partial f}{\partial \eta} g(\eta)-K\left[2 g(\eta)+\frac{\partial f^{2}}{\partial \eta}\right]=0 \\
\frac{\partial \theta^{2}}{\partial \eta}+\operatorname{Pr} f(\eta) \frac{\partial \theta}{\partial \eta}=0
\end{gathered}
$$

subjected to the boundary conditions

$$
\begin{gathered}
f=0, \quad \frac{\partial f}{\partial \eta}=1+k \frac{\partial^{2} f}{\partial \eta^{2}}, \quad g=-n \frac{\partial^{2} f}{\partial \eta^{2}}, \quad \theta=1, \text { at } \eta=0, \\
\frac{\partial f}{\partial \eta}=\delta, \quad \frac{\partial^{2} f}{\partial \eta^{2}}=0, \quad g=0, \quad \theta=0 \text { as } \eta \rightarrow \infty,
\end{gathered}
$$

where $\beta$ is the Deborah number, $\delta$ is the stretching stretch parameter, $k$ is velocity slip parameter and Pr is the Prandtl number which are defined as

$$
\beta=\lambda_{2} c, \delta=\frac{a}{c}, k=g_{x} \sqrt{\frac{c\left(1+\lambda_{1}\right)}{\nu}}, \operatorname{Pr}=\frac{\mu c_{p}}{k} .
$$

The physical quantities of principal interest such as the skin friction coefficient $C_{f}$ and the local Nusselt number $N u_{x}$ are defined as

$$
C_{f}=\frac{\tau_{w}(x)}{\rho u_{w}^{2}} \text { and } N u_{x}=\frac{q_{w}(x) x}{k\left(T_{w}-T_{\infty}\right)}
$$

where the $\tau_{w}(x)$ is the shear stress at the wall and $q_{w}(x)$ is the heat flux from the surface of the sheet, are given as

$$
\tau_{w}(x)=\left([\mu+\kappa] \frac{\partial u}{\partial y}+\kappa N\right)_{y=0} \text { and } q_{w}(x)=-k\left(\frac{\partial T}{\partial y}\right)_{y=0} .
$$

Using variables (9), we get

$$
C_{f} \operatorname{Re}_{x}^{1 / 2}=[1+(1-n) K] \frac{\partial^{2} f}{\partial \eta^{2}}(\tau, 0), \frac{N u_{x}}{\operatorname{Re}_{x}^{1 / 2}}=-\frac{\partial \theta}{\partial \eta}(\tau, 0) .
$$

where $R e_{x}$ is the Reynolds number.

\section{RESULT AND DISCUSSION}

Equations (10) to (12) subject to the boundary conditions (13) were solved numerically using an implicit finite difference method known as Keller box. This method has been found to be very suitable in dealing with nonlinear parabolic problems and practically used by many researchers [29-30, 35-36]. The effects of Prandtl number Pr, material $K$, velocity slip $k$, Deborah number $\beta$ and stretching 
parameters $\delta$ are analyzed for the viscous and incompressible micropolar Jeffrey fluids. The present results for the heat transfer coefficient, $-\theta^{\prime}(0)$ with various values of Prandtl number Pr are compared with those in Turkyilmazoglu and Pop [19] for the limiting cases without the influence of material parameter $K$, concentration of microelements $n$ and velocity slip $k$ parameter at the fixed values of Deborah number and stretching parameter in order to validate the numerical results. It is seen from Table 1, the obtained numerical results using the proposed numerical method are in an excellent agreement with those published previously.

TABLE 1. Comparison the values of heat transfer coefficient $-\theta^{\prime}(0)$ at $K=0, k=0, n=0, \beta=2, \delta=1$

\begin{tabular}{|c|c|c|}
\hline $\mathbf{P r}$ & Turkyilmazoglu and Pop [19] & Present \\
\hline 2 & 1.12837917 & 1.128732 \\
\hline 5 & 1.78412412 & 1.785519 \\
\hline 10 & 2.52313252 & 2.527078 \\
\hline
\end{tabular}

Figure 1 depicts the influence of Prandtl number on the temperature profile $\theta(\eta)$. It is observed that, the temperature profiles rapidly decreases with the increasing values of Prandtl number. This behaviour is expected due to the fact that for the smaller values of $\mathrm{Pr}$, fluid possesses high thermal conductivity and heat diffuses away from the surface faster than at the higher values of Pr. Thus, the boundary layer becomes thinner and consequently decreases the temperature when Pr is increased. It is obvious that, a large Prandtl number is important for non-Newtonian fluid however, from the figure, an identical behaviour is observed on the temperature profile regardless of whether the Prandtl number is small or large. Therefore, the values of Prandtl number, $\operatorname{Pr}$ is fixed to unity as $\operatorname{Pr}=0.71$ for all figures in this problem.

Figures 2 until 7 represent the distribution of velocity $f^{\prime}(\eta)$, microrotation $g(\eta)$ and temperature $\theta(\eta)$ at a fixed value of $n=0$ for different values of the physical parameters of interest. The case $n=0$ corresponds to the boundary condition indicating the no spin condition, which is happens when the particle density is sufficiently large, so that the microelements close to the wall are not able to rotate as stated by Jena and Mathur [31]. Figures 2 to 4 show the distribution of velocity, microrotation and temperature for different values of Deborah number. It can be seen that, the velocity increased when the values of Deborah number are increased whereas the opposite behaviour is noticed for the temperature profile. The fact that the larger value of Deborah number corresponds to the higher relaxation time and decrease the values of temperature. It is also observed that the microrotation profile decreases near the sheet but twist the pattern between $1.5<\eta<2.5$ where the profiles start to increase and become zero far away from the sheet. These results 


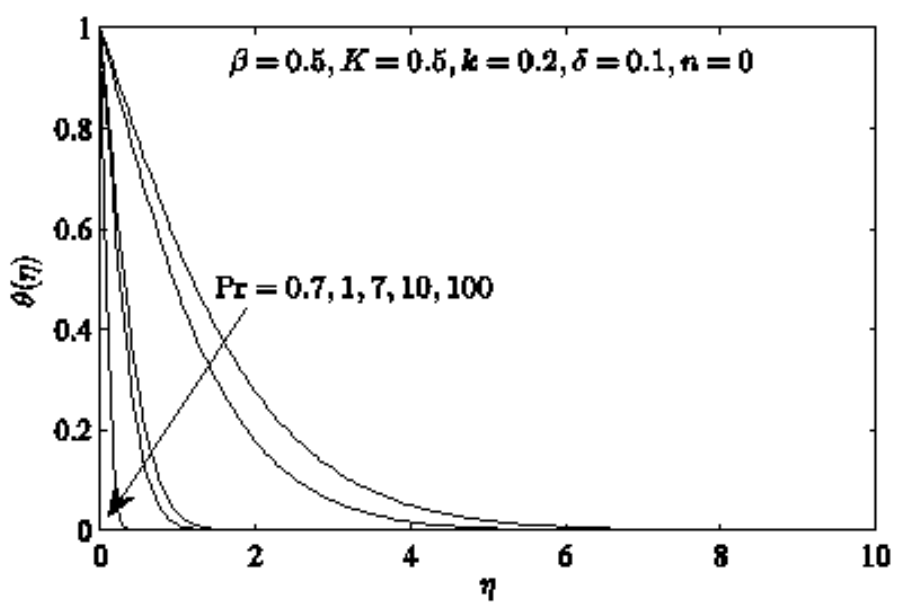

FiguRE 1. The temperature profiles for various values of $\operatorname{Pr}$ when $n=0$.

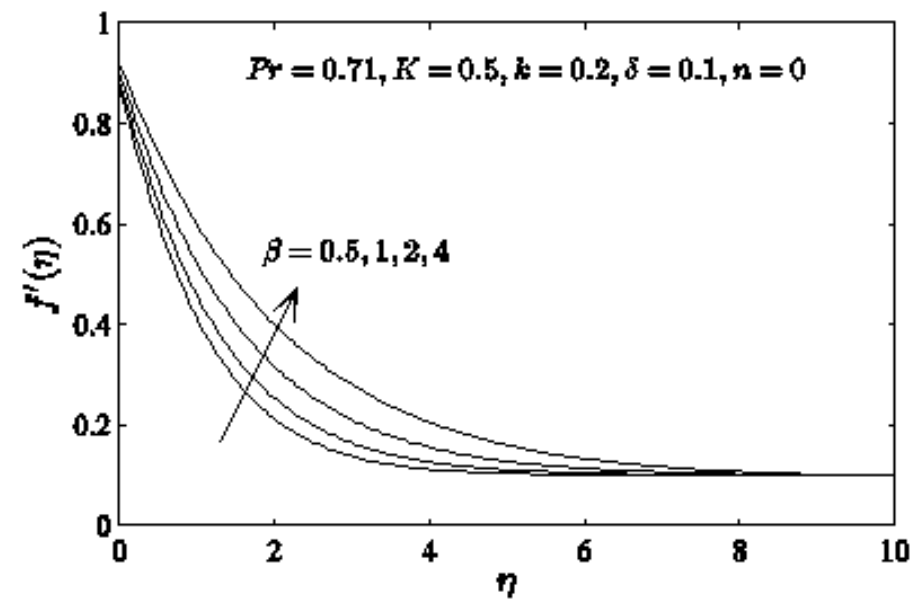

Figure 2. The velocity profiles for various values of $\beta$.

also support the computation for this investigation since it satisfies the boundary condition applied.

The effects of material parameter $K$ on the velocity $f^{\prime}(\eta)$, microrotation $g(\eta)$ and temperature profiles $\theta(\eta)$ are illustrated in figures 5 to 7 . It is observed that, an increase of $K$, leads to decrease the velocity of fluid while it increases the rate of temperature. Meanwhile, figure 6 depicted the rate of microrotation where it 
Numerical Solutions For Convective Boundary Layer Flow Of Micropolar Jeffrey Fluid 293

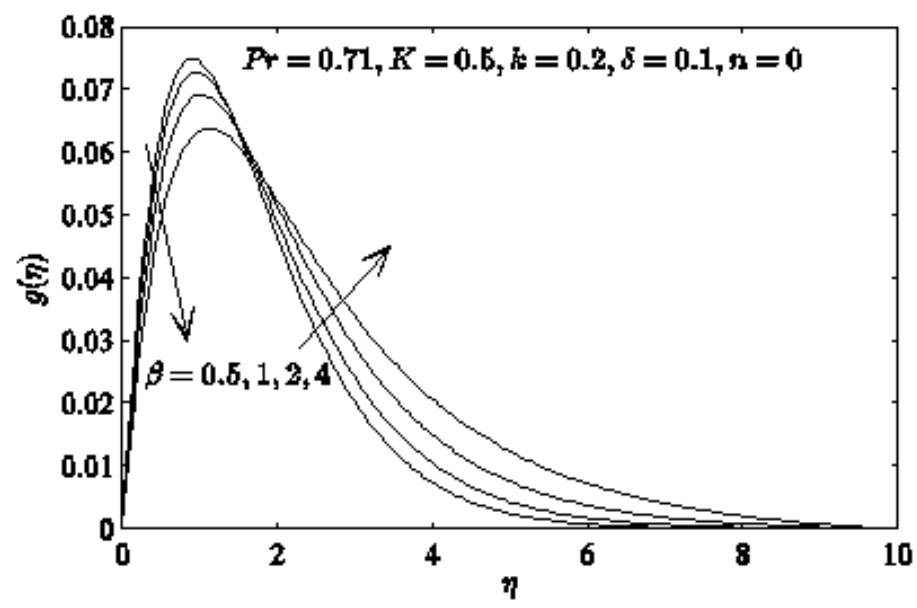

FIguRE 3. The microrotation profiles for various values of $\beta$.

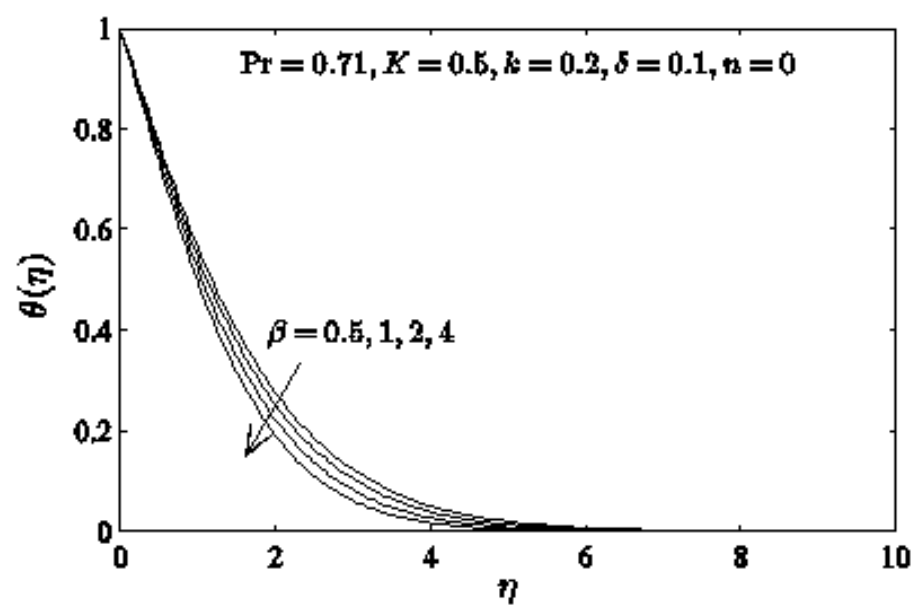

Figure 4. The temperature profiles for various values of $\beta$.

is increases gradually with $K$ until one maximum value and later slowly decreases until asymptotically zero.

The influence of Deborah number $\beta$ on the variation of heat transfer coefficient $-\theta^{\prime}(0)$ with Pr for both cases, strong $(n=0)$ and weak $(n=0.5)$ concentrations is shown in figure 8 . It is noticed that, when the values of $\beta$ increase, its lead to decrease the values of $-\theta^{\prime}(0)$ but the contradict behavior is noticed for the increment of the values of Pr. From these results, it can be concluded that, 


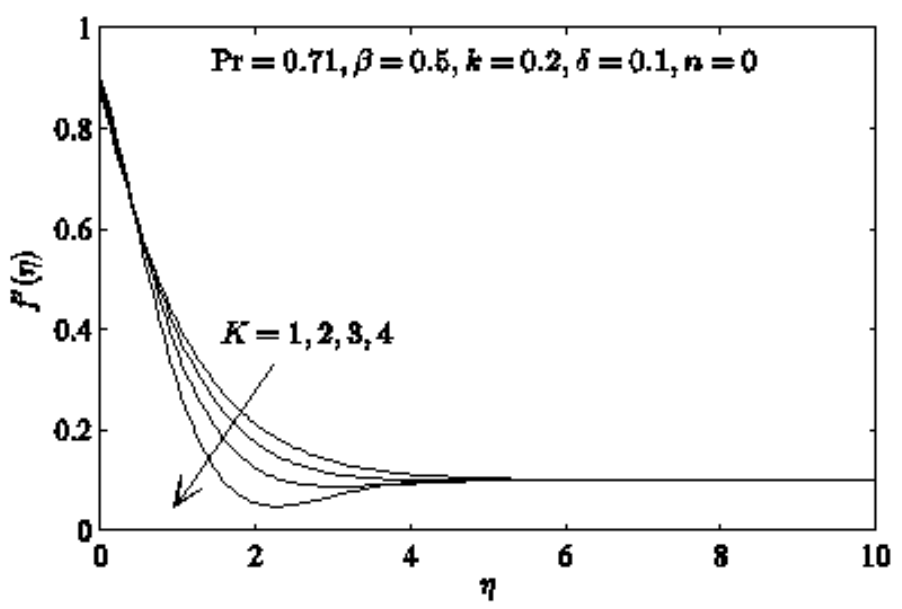

Figure 5. The velocity profile for various values of $K$.

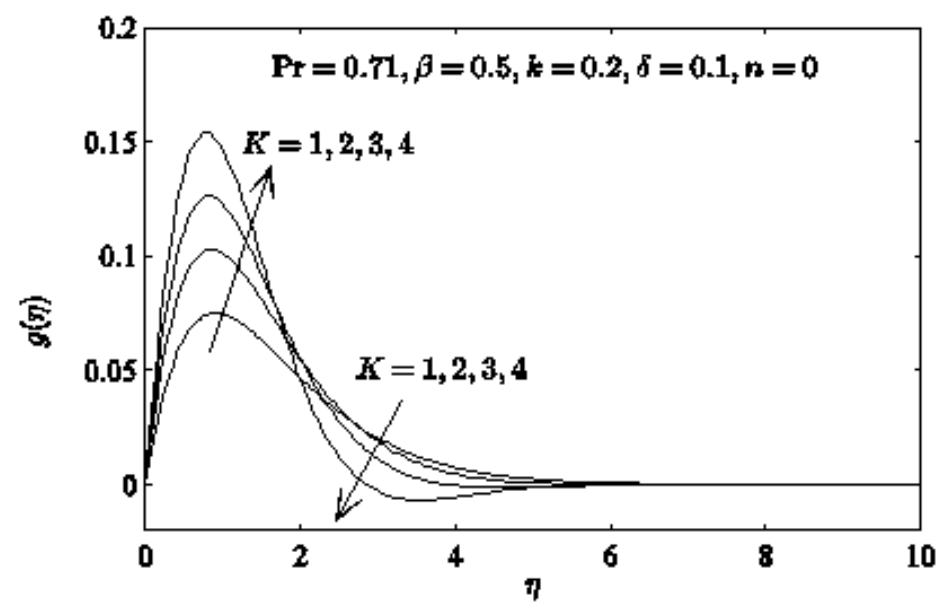

FiguRE 6. The microrotation profile for various values of $K$.

the Deborah number retarded the heat transfer of fluid while the values of Prandtl number boosted the transfer of heat.

Table 2 presented the rate of reduced skin friction $f^{\prime \prime}(0)$ and heat transfer coefficient $-\theta^{\prime}(0)$ with various values of studied parameter. As values of Deborah number and stretching parameter increased, both values of skin friction and heat transfer coefficient. Besides that, it is also noticed that, the increment values in the velocity slip and material parameters led to boost the rate of skin friction and reduce the rate of heat transfer coefficient. 


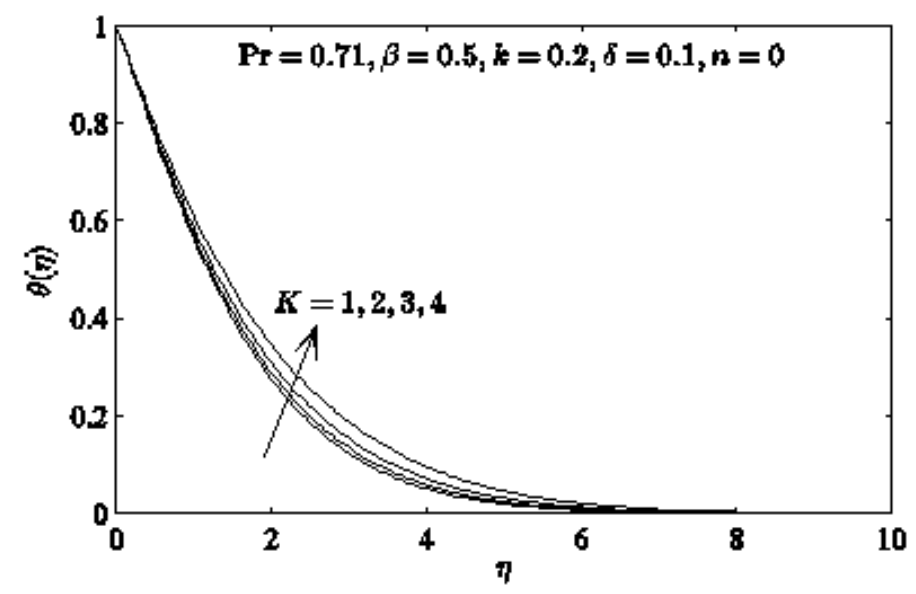

Figure 7 . The temperature profile for various values of $K$.

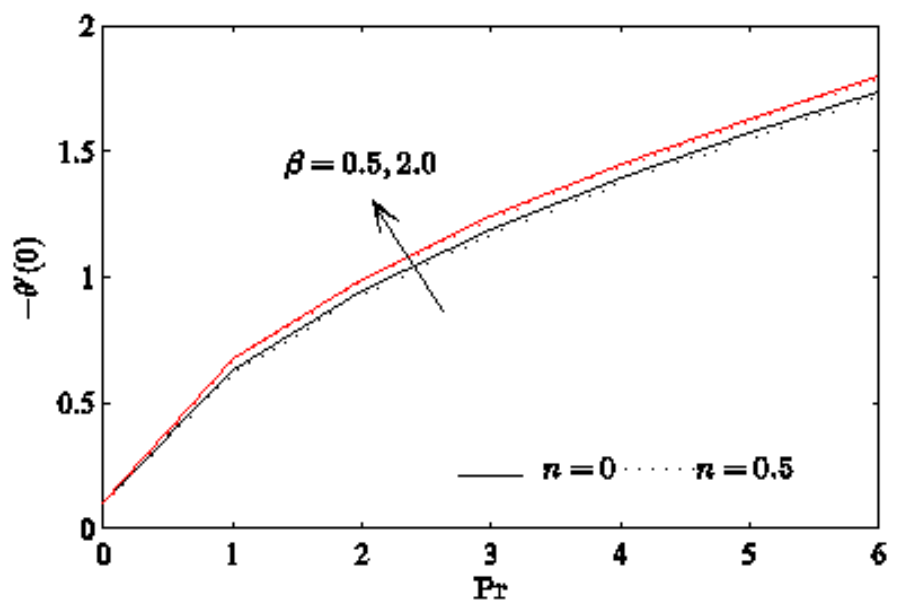

Figure 8. Distribution of $-\theta^{\prime}(0)$ with various values of $\beta, \operatorname{Pr}$ and $n$.

\section{CONCLUSION}

In this paper, the convective boundary layer flow of micropolar Jeffrey fluid past a permeable stretching sheet has been investigated. The governing partial differential equation of respective problem are first transformed into ordinary differential equation using appropriates similarity transformation. The results have been computed using Keller box method. From investigations, results clearly show that the Prandtl number, Deborah number, and material parameter for the prescribe 
TABLE 2. The values of reduced skin friction $f^{\prime \prime}(0)$ and heat transfer coefficient $-\theta^{\prime}(0)$ with various parameters at $n=0$ and $\operatorname{Pr}=$ 0.71

\begin{tabular}{|c|c|c|c|c|c|}
\hline$\beta$ & $K$ & $\delta$ & $k$ & $f^{\prime \prime}(0)$ & $-\theta^{\prime}(0)$ \\
\hline 0.5 & 0.5 & 0.2 & 0.1 & -0.675724 & 0.512421 \\
\hline 1.0 & & & & -0.601075 & 0.528987 \\
\hline 2.0 & & & & -0.505441 & 0.550805 \\
\hline 4.0 & & & & -0.402150 & 0.574847 \\
\hline 6.0 & & & & -0.344527 & 0.588323 \\
\hline 0.5 & 0.2 & & & -0.682562 & 0.513064 \\
\hline & 0.3 & & & -0.680675 & 0.512893 \\
\hline & 0.5 & & & -0.675724 & 0.512421 \\
\hline & & 0.2 & & -0.675724 & 0.512421 \\
\hline & & 0.5 & & -0.501184 & 0.574805 \\
\hline & & 1.0 & & 0.000000 & 0.672384 \\
\hline & & 2.0 & & 1.598869 & 0.841754 \\
\hline & & 5.0 & & 9.074552 & 1.225625 \\
\hline & & & 0.1 & -0.675724 & 0.512421 \\
\hline & & & 0.5 & -0.489112 & 0.466707 \\
\hline & & & 0.8 & -0.407196 & 0.444855 \\
\hline & & & 1.0 & -0.366811 & 0.433568 \\
\hline
\end{tabular}

wall temperature together with strong concentration of microelements microrotation $(n=0)$ affect the fluid flow characteristics in term of velocity, temperature, microrotation as well as the heat transfer of the fluid.

Acknowledgement. The authors would like to acknowledge Ministry of Education (MOE) and Research Management Centre-UTM for the financial support through vote numbers 5F004, 07G75,07G70 and 07G76 for this research. 
Numerical Solutions For Convective Boundary Layer Flow Of Micropolar Jeffrey Fluid 297

\section{REFERENCES}

[1] Eldabe, N.T., Moatimid, G.M. and Ali, H.S., Magnetohydrodynamic flow of non-Newtonian visco-elastic fluid through a porous medium near an accelerated plate, Can. J. Phys., 81 (2003), 1249-1269.

[2] Hameed, M. and Nadeem, S., Unsteady MHD flow of a non-Newtonian fluid on a porous plate, J. Math. Anal. Appl., 325 (2007), 724-733.

[3] Abel, M.S., Siddheshwar, P.G. and Nandeppanavar, M.M.,vHeat transfer in a viscoelastic boundary layer flow over a stretching sheet with viscous dissipation and non-uniform heat source, Int. J. Heat Mass Transfer, 50 (2007), 960-966.

[4] Das, K., Slip effects on MHD mixed convection stagnation point flow of a micropolar fluid towards a shrinking vertical sheet, Com. Math. Appl., 63 (2012), 255-267.

[5] Kasim, A.R.M., Mohammad, N.F. , Aurangzaib and Shafie, S., Natural convection boundary layer flow of a viscoelastic fluid on solid sphere with Newtonian heating, World Academy Sc. Eng. Tech., 64 (2012), 628-633.

[6] Ali, F., Khan, I. and Shafie, S., Closed form solutions for unsteady free convection flow of a second grade fluid over an oscillating vertical plate, PloS one, 9 (2014), 1-15.

[7] Khan, I., Ali, F., Shafie, S. and Qasim, M., Unsteady free convection flow in a Walters-B fluid and heat transfer analysis, Bull. Malays. Math. Sci. Soc., 37 (2014), 437-448.

[8] Ahmad, S., Vieru, D., Ilyas, I. and Shafie, S., Unsteady magnetohydrodynamic free convection flow of a second grade fluid in a porous medium with ramped wall temperature, PloS one, 9 (2014), 1-9.

[9] Zaib, A. and Shafie, S., Slip effect on an Unsteady MHD stagnation-point flow of a micropolar fluid towards a shrinking sheet with thermophoresis effect, Int. J. Comput. Methods Eng. Sci. Mech., 16 (2015), 285-291.

[10] Khalid, A., Khan, I. and Shafie, S., Unsteady boundary layer flow of a Casson fluid past an oscillating vertical plate with constant wall temperature, Malaysian J. Fundam. Appl. Sci., 11 (2015), 28-32.

[11] Bird, R.B., Armstrong, R.C. and Hassager, O., Dynamics of polymeric liquids, John Wiley \& Sons, 1987.

[12] Jeffreys, H., The Earth, Cambridge University Press, London, 1929

[13] Nadeem, S. and Akbar, N.S., Peristaltic flow of a Jeffrey fluid with variable viscosity in an asymmetric channel, Z Naturforsch. A: Phys. Sci., 64 (2009), 713-722.

[14] Khan, M., Iftikhar, F. and Anjum, A., Some unsteady flows of a Jeffrey fluid between two side walls over a plane wall. Z Naturforsch. A: Phys. Sci., 66 (2011), 745-752.

[15] Hayat, T., Asad, S., Qasim, M. and Hendi, A.A., Boundary layer flow of a Jeffrey fluid with convective boundary conditions, Int. J. Numer. Methods Fluids, 69 (2012), 1350-1362.

[16] Hayat, T., Shehzad, S.A., Qasim, M. and Obaidat, S., Thermal radiation effects on the mixed convection stagnation-point flow in a Jeffery fluid, Z Naturforsch. A: Phys. Sci., 66 (2011), 606-614.

[17] Hayat, T., Ahmad, N. and Ali, N., Effects of an endoscope and magnetic field on the peristalsis involving Jeffrey fluid, Commun. Nonlinear Sci. Numer. Simul., 13 (2008), 1581-1591.

[18] Vajravelu, K., Sreenadh, S. and Lakshminarayana, P., The influence of heat transfer on peristaltic transport of a Jeffrey fluid in a vertical porous stratum, Commun. Nonlinear Sci. Numer. Simul., 16 (2011), 3107-3125.

[19] Turkyilmazoglu, M. and Pop, I., Exact analytical solutions for the flow and heat transfer near the stagnation point on a stretching/shrinking sheet in a Jeffrey fluid, Int. J. Heat Mass Transfer, 57 (2013), 82-88.

[20] Zin, N.A.M., Ilyas, I. and Shafie, S., Exact and numerical solutions for unsteady heat and mass transfer problem of Jeffrey fluid with MHD and Newtonian heating effects, Neural Comput. Appl., (2017), 1-17.

[21] Eringen, A.C., Theory of micropolar fluids, J. Math. Mech., 16 (1966).

[22] Gorla, R.S.R., Micropolar boundary layer flow at a stagnation point on a moving wall, Int. J. Eng. Sci., 21 (1983), 25-33. 
[23] Agarwal, R.S. and Dhanapal, C., Flow and heat transfer in a micropolar fluid past a flat plate with suction and heat sources, Int. J. Eng. Sci., 26 (1988), 1257-1266.

[24] Nazar, R. and Amin, N., Free convection boundary layer on an isothermal sphere in a micropolar fluid, Int. Commun. Heat Mass Transfer, 29 (2002), 377-386.

[25] El-Arabawy, H.A., Effect of suction/injection on the flow of a micropolar fluid past a continuously moving plate in the presence of radiation, Int. J. Heat Mass Transfer, 46 (2003), 1471-1477.

[26] Ishak, A., Nazar, R. and Pop, I., Heat transfer over a stretching surface with variable heat flux in micropolar fluids, Phys. Lett. A, 372 (2008), 559-561.

[27] Cheng, C.Y., Natural convection heat and mass transfer from a sphere in micropolar fluids with constant wall temperature and concentration, Int. Commun. Heat Mass Transfer, 35 (2008), 750-755.

[28] Nadeem, S., Hussain, M. and Naz. M., MHD stagnation flow of a micropolar fluid through a porous medium, Meccanica, 45 (2010), 869-880.

[29] Aurangzaib, Kasim, A.R.M., Mohammad, N.F. and Shafie, S., Unsteady MHD mixed convection flow with heat and mass transfer over a vertical plate in a micropolar fluid-saturated porous medium, J. Appl. Sci. Eng., 16 (2013), 141-150.

[30] Zaib, A. and Shafie, S., Slip Effect on an Unsteady MHD Stagnation-Point Flow of a Micropolar Fluid towards a Shrinking Sheet with Thermophoresis Effect, Int. J. Comput. Methods Eng. Sci. Mech., 16 (2015), 285-291.

[31] Jena, S.K. and Mathur, M.N., Similarity solutions for laminar free convection flow of a thermomicropolar fluid past a non-isothermal vertical flat plate, Int. J. Eng. Sci., 19 (1981), 1431-1439.

[32] Ahmadi, G., Self-similar solution of incompressible micropolar boundary layer flow over a semi-infinite plate, Int. J. Eng. Sci., 14 (1976), 639-646.

[33] Rees, D.A.S. and Bassom, A.P., The Blasius boundary-layer flow of a micropolar fluid, Int. J. Eng. Sci., 34 (1996), 113-124.

[34] Lok, Y.Y., Amin, N., Campean, D. and Pop, I., Steady mixed convection flow of a micropolar fluid near the stagnation point on a vertical surface, Int. J. Numer. Methods Heat Fluid Flow, 15 (2005), 654-670.

[35] Rawi, N.A., Kasim, A.R.M., Isa, M. and Shafie, S. Shafie, g-Jitter induced mixed convection flow of heat and mass transfer past an inclined stretching sheet, Jurnal Teknologi, 71 (2014), 27-31.

[36] Kasim, A.R.M., Jiann, L.Y., Rawi, N.A., Ali, A. and Shafie, S., Mixed Convection Flow of Viscoelastic Fluid over a Sphere under Convective Boundary Condition Embedded in Porous Medium, Defect Diffusion Forum, 362 (2015), 67-75. 